R. Ilalianistica, Sâu Paulo, ano II, n² 2, p. 113-119, 1994.

\title{
LE MOSCHE DEL CAPITALE DI PAOLO VOLPONI.
}

\author{
Lucia Wataghin*
}

\begin{abstract}
Resumo: Em Le mosche del capliale Volponi analisa as re óes entre o poxder do capital e o mundo industrial. O romancista acredita que a literalura possa exece 'Ina influência subre a luta de classe.
\end{abstract}

Palavras-chave: romance político, capital ismo e indústria, narrativa contemporânea.

"Ad essere onesto, cioè essenziale, come scrittore, credo che mi conducano la mia passione morale ed anche la mia vocazione politica" ${ }^{\prime \prime}$

Alla radice della vocazione letteraria di Volponi c'è, prima di tutto, una passione etico/politica che fa della letteratura uno strumento d'intervento sulla società, ovvero che afferma la funzione della cultura, e quindi della letteratura, come politica. Il rapporto con la fabbrica, in cui lo scrittore ha lavorato dal 1950 fino all'inizio degli anni Settanta, dedicandosi essenzialmente ai problemi dell'organizzazione e dell'assistenza al personale, è fondamentale nella formazione della visione del mondo di Volponi. Con Le mosche del capitale, pubblicato da Einaudi nel 1989, Paolo Volponi torna a questo tema, che fu anche il tema del suo primo romanzo, Memoriale, del 1962. Scrivendo a proposito di Memoriale, Volponi affermava:

"Sono stato aiutato, o forse è meglio dire mosso, dalla pena del mio lavoro quotidiano in una grande fabbrica, toccato dai problemi di un mondo in convulsione, come è quello industriale, traboccante $e$ incandescente, che cerca di correre dietro al progresso scientifico portandosi dietro un grosso bagaglio medievale ${ }^{2}$

\footnotetext{
- Professora de Língua e Literalura ltaliana junto no Departamento de Letras Modernas da Faculdade de Filosofia, Letras e C'iências llumanas, USP.

1 Volponi, Pnolo, "Notizia autubiografica", in G. C. Ferretti, "Officluna" cultura, lelleratura e politica degli auni cinquanta, Einaudi, Torino, 1975, p. 80-81 
WATAghiN, Lucia. Le mosche del capliale di Paolo Volponi.

Lo stesso conflitto, fra una valutazione positiva e ottimista delle scoperte scientifiche come possibile soluzione dei problemi dell'umanità, e la denuncia dell'uso sconsiderato che ne fa il capitale, col suo pesante retaggio di arretratezza culturale e politica, diventa il tema delle Mosche del capitale. In questo libro in particolare è descritto il conflitto fra la potenzialità immensamente benefica della fabbrica, non solo come produttrice di beni per l'umanità, ma come "un nuovo modo di lavorare $e$ forse addiritura un nuovo luogo, meno confuso, da cui vedere il mondo", e la gestione della fabbrica, affidata ad una classe sociale (le mosche del capitale) legata ad una visione del mondo medieval-fascista, e anche per questo assolutamente incapace di proporre un ordine sociale accettabile.

Alla contraddizione messa in luce da Volponi fra il mondo moderno e la realtà sociale arretrata allude Calvino, in un elogio dell'opera di Volponi:

"La tensione lirico-trasfigurativa che Volponi raggiunge risulta essere la più adatta a esprimere la contradditoria e provvisoria realtà attuale: fra tecniche produttive avanzate e situazione social-antropologica arretrata, tra fabbriche tutte vetri acciaio human relations e un'Lalia oscuramente biologica" ${ }^{3}$

In questo quadro, era prevedibile che la critica marxista individuasse in Volponi, fin dall'inizio della sua carriera (anche se con fortune alterne presso i critici), come uno dei primi scrittori italiani (insieme a Pasolini e Roversi, fra gli altri) come uno dei primi scrittori italiani contemporanei che si propongono di vivere gli scontri con "una realtà sempre pin difficile" ${ }^{4} \mathrm{e}$ quindi, come scrisse ancora Calvino, uno dei primi "a modificare il corso elegiaco-moderato-sociologico del romanzo italiano".

Nettamente politico, più di tutti i romanzi che lo precedono, è quest'ultimo, Le mosche del capitale. La vicenda del libro è unitaria, relativamente semplice (se paragonata per esempio a Corporale), e si concentra sul complesso dei rapporti fra coloro che rappresentano il potere e un singolo volenteroso che misura l'impossibilità di anche solo scalfire l'ordine sociale stabilito. Quello che interessa non è tanto il tormento interiore del protagonista (il cui nome di battesimo è Bruto, un'allusione

3 Da una recensione apparsa su L'tllustrazhome liallana, maggio 1962; cit. da Enrico Baldise, Invilo alta kettura di Volponi, Mursia, Milano, 1982, p. 81.

4

Iblem, p. 61. 
forse alla contraddizione implicita nella sua posizione di beniamino del potere, posizione da cui si ritira quando sono sconfitti i suoi tentalivi di riforme), ma piuttosto le fila, i meccanismi, le trame di tutto quel grande gioco di cui Volponi denuncia con precisione le maniere e l'inesorabilità: l'oscura potenza e l'invincibile volontà di auto-perpetuazione dell'organismo che sta già al potere.

Il protagonista del romanzo è un alto dirigente dell'industria, che possiamo definire un alter ego dello scrittore, nella misura in cui la sua vicenda professionale e la sua sinificativa scelta finale coincidono massicciamente con quella dello stesso Volponi.

Di tutti i protagonisti dei romanzi di Volponi, Saraccini è forse l'unico sostanzialmente "sano", perfetlamente integrato nell'industria, e nella società. Il suo rapporto con l'industria è inizialmente non antagonista, fiducioso, non irritato, anzi, pieno di ammirazione, speranza, un rapporto quasi ingenuo con il potere. Su tale ingenuità, Volponi comincia a costruire il quadro delle relazioni degli uomini al potere con i subordinati più prossimi, fondandone il discorso su parole-chiave che rimandano al medioevo (come devozione, sudditi fedeli, regno, nobiltì, ecc.).

Per completare il quadro ideologico, Volponi allude al retaggio del fascismo nell'industria moderna, non solo nel discorso, ma nelle persone fisiche riammesse ai vertici dell'industria o in loro dipendenti, nonostante il passato al servizio del governo fascista.

Sotto accusa non è la fabbrica, ma è direttamente la classe al potere. E' ben chiara, nel romanzo, la distinzione fra capitale e industria. Nelle prime pagine del romanzo Saraccini formula un paragone fra Mozart e suo padre da una parte, e il capitale e l'industria dall'altra. Si tratta del controllo, avido e spietato, da parte dell'impresa (paragonata a Mozart padre) sull'immensa potenzialità benefica e rivoluzionaria, il genio, dell'industria (Mozart figlio). Il romanzo tratta del conflitto fra la potenzialità, che Volponi considera immensamente benefica, della fabbrica e la sua effelliva realizzazione, per mano della classe che sta al potere.

La vicenda di Saraccini è praticamente identica a quella di Volponi: entrambi sono capi del personale di una grande industria, e ad un certo punto della carriera ricevono l'offerta dell'altissima carica di amministratore delegato. Rifiutano, e fanno una controproposta, di riforme significative nell'amministrazione, e di apertura radicale alle esigenze dei lavoratori. La controproposta è rifiutata, e devono dimettersi. Di fronte alla certezza che il suo progetto è destinato al fallimento, Saraccini si ripromette di scrivere un libro bianco sulla fabbrica, o meglio, sulle mosche del capitale: 
WATAgilin, I ucia. Le mosche del capltale di Paulo Volponi.

"Un giorno dirò tutto, scriverò un memoriale, un libro bianco sui grandi dirigenti, sulle grandi politiche aziendali, la verità sulla ricerca e lo sviluppo, sulle qualità produttive, sugli investimenti, sulle grandi novità tecnologiche, sui grandi, questi si, altro che grandi, prelievi personali e soprusi, sulle mosche, si, le mosche del capitale.

Si fermò su questa immagine, che gli pareva cogliesse esattamente la banda dei suoi nemici, tutti gli amministratori e $i$ manager industriali di successo, fatti di voli e voletti, di ali $e$ alette... azzurre come cravatte... tutti a modo, con gesti $e$ accenti, aggiornamenti e riverenze, relazioni e riferimenti, le sapienti colorate voraci mosche del capitale, sì, le mosche... per di più svolazzano e ronzano dappertutto, in bell'inglese, per andare a succhiare e a sporcare"

Il centro del romanzo si definisce così non tanto intorno alla vicenda personale e professionale di Saraccini, o degli altri personaggi, ma piuttosto come la fitta trama dei rapporti del potere. Il vero protagonista del romanzo è il potere, come dice lo stesso Saraccini, quando si accorge di non avere nessuna possibilità di realizzare il proprio progetto:

"Non ci sono più personaggi perché nessuno agisce come tale, nessuno ha un proprio copione. L'unico personaggio, è banale dirlo, è il potere"ro.

Nel descrivere Saraccini, Volponi ha rinunciato a dargli uno spessore psicologico, una vita qualsiasi al di fuori della sua storia nella fabbrica. Ha rinunciato alla "corporalità", alle nevrosi, agli amori:

"Oggi che cosa può interessare dei miei amori, a chi? A chi può interessare sapere le mie rapine sessuali? A chi sapere come era organizzata la mia vita privata, arredata la mia casa, $i$ libri che leggevo?"7

5 Volponi, Paulo, Le nosclse del capilsle. Torino, Einaudi, 1989, p. 132-133.

$6 \quad$ Ibidem, p. 137.

7 Ibidem, p. 137. 
R. Italianistica, Sño Paulo, ano II, n² 2, p. 113-119, 1994.

Nel romanzo appaiono personaggi-oggetti, la borsa del presidente, le tende, i mobili, la penna, la poltrona, i ficus, le porte, ecc., tutti a rappresentare sfumature del potere, qualità, idee, e anche effettivi movimenti, a raccontare cioè la storia dal punto di vista del particolare aspetto del potere che rappresentano. Le qualità umane sono meglio rappresentate dagli oggetti che dalle persone, quando sono ridotte all'estremo della compiacenza, del servilismo, della devozione, soltomissione, negazione della libertà dell'vomo. Tutta la trama dei rapporti del potere, con i suoi personaggi, umani e non, è caratterizzata dall'alienazione, e più specificamente dalla spersonalizzazione. E' descritta la psicologia del potere, e quindi la psicologia dei , rsonaggi non in quanto uomini, ma solo nella misura in cui sono legati al pe 'те, solo strettamente nei loro rapporti con il potere. Tutto confluisce intorno a questa idea del potere, con le sue manovre (descritte con la fantastica precisione di $L$. che le ha conosciute profondamente, di persona), col suo linguaggio nauseante, sempre tendenzioso, ipocrita, barocco, gonfio, sempre immorale, maneggione, ambiguo, in cui non si distingue più (nel parlato e perfino nel pensiero intimo dei personaggi, non espresso ad altri) la parola sincera dalla manovra, dall'adulazione, dalla strumentalizzazione sistematica delle cose e delle persone. Anche l'universo sottostante i palazzi dell'industria, la città, i drammi personali dei suoi abitanti, e degli operai dell'industria, che sono la conseguenza storica dell'amministrazione dell'industria, tutto confluisce a comporre il quadro il cui centro è il potere. Del resto, è interessante notare che nella classe operaia e nel resto della città Volponi torna a riconoscere una umanità, certamente meno alienata, capace di capire, di sentire e di soffrire, $\mathrm{e}$ in fin dei conti più libera rispetto alle mosche del capitale:

\section{"La cittì si è fatta aggriccita di venuzze e infezioni. Gli operai dell'industria lo notano e lo patiscono più di tutti."}

D'altra parte, il destino, e la stessa identità dell'operaio si confondono con quello della fabbrica, a cui secondo Volponi non si vuole e non si deve rinunciare. Per l'operaio Tecraso, la fabbrica è un modo di vita, una scelta ordinatrice, una visione "meno confusa" del mondo: In fabbrica ci si capisce di più, e si può guardar fuori..." Tecraso, nella lettera all'azienda in cui reagisce al licenziamento, scrive:

\footnotetext{
8 Ibidem, p. 192.

9 Ibidem, p. 19.
} 
Wataghin, Lucia. Le musche del capilale di Paulo Volponi.

"P.S. Se non riceverò una vostra lettera, anche soltanto interlocutoria, entro giorni 20, mi riterrò libero di decidere da solo della mia vita, quella grande che vi riguarda e quella mia secondaria" $^{10}$

La lingua del romanzo è ricchissima, allucinante, iperbolica, immaginosa, barocca, espressionista con accenti gaddiani, spesso lirica, ellittica, piena di anacoluti, neologismi, anglicismi, termini appartenenti al linguaggio settoriale dell'industria e dell'universo manageriale, che si confondono spesso col linguaggio sindacale e operaista; una lingua che ha incorporato criticamente la logica delle relazioni dell'industria e che ne riflette lo spirito, attingendo alla fonte del linguaggio pragmatico e ridondante caralteristico dei rapporti potere/sindacato/operai/individui ("iniziative, rapporti, confronti") ${ }^{11}$, delle spiegazioni, delle lotte, dei discorsi. Una lingua che ha ironicamente incorporato la logica dell'efficienza, delle esigenze dell'industria, una lingua integralmente contaminata, caratterizzata dallo svuotamento del significato delle parole al di fuori del loro uso industriale, dall'impolenza, dalla banalizzazione: "I sindacalisti si offesero, si opposero, condannarono, si riunirono"12 $\mathrm{La}$ ricchezza, l'eccesso, la banalizzazione, l'abuso delle parole sottolinea senz'altro da una parte il caos insito in questi rapporti, dall'altra l'impotenza del discorso di fronte alla realtà del potere. Il che del resto è detto esplicitamente, a proposito per esempio di Saraccini:

"Lui aveva davero l'impressione di camminare, vivere $e$ lavorare soltanto sulle parole, sui termini di relazioni, dichiarazioni, principi, ordini e organigrammi"13 ; "Saraccini è molto confuso, non riconosce più la sua posizione di giudizio, di iniziative, rapporti, confronti"14 ; "L'italiano pare una lingua ormai fatta e usata solo per ingannare togliere truffare rubare $^{15}$

10 Ibidem, p. 230 (suttolineatura mia).

11 Ibidem, p. 243.

12 Ibidem, p. 227.

13 lbidem, p. 216.

14 lbidem, p. 243.

15 Ibiden, p. 191. 
Le mosche del capitale si configura così, a livello linguistico come a livello di contenuto, come rivolta, come denuncia, come innovazione, e insomma come strumento critico, d'intervento sulla realtà. Anche la conclusione del romanzo conferma l'esistenza di una tesi politica, a cui è rimandata l'effettiva soluzione del dramma. Saraccini, nel suo discorso di rinuncia definitiva al suo progetto, profelizza al presidente lo scontro inevilabile con i sudditi e la fine del regno. L'ultima scena del romanzo descrive la morte del presidente, morte per un cancro che ha la stessa fisiologia e fisionomia del malato. Il presidente sarà immediatamente sostituito da un uomo-forte dell'azienda, dotato della sua stessa logica. Ma il cancro del morente suona come l'avvertimento di an analogo cancro che corrode il potere dall'interno.

\section{BIBLIOGRAFI CONSULTATA}

VOL.PONI, Pnolo. Le mosche del cupitale. Torino, Einaudi, 1989.

FERRETTI, G. C. "Volponi: la "diversità" come eversione", in: La letteratura del rifiuto e altri scritti sulla crisi e trasformazione dei ruoli intellettuali. Milano, Mursia, 1968-1981.

FERRETTI, G.C. Paolo Volponi. Firenze, La Nuova Italia, 1972.

BAL.DISE, Enrico. Invito alla lettura di Volponi. Milano, Mursia, 1982.

CAMON, Ferdinando. Il mestiere di scrittore. Conversazioni critiche con Bassani, Calvino, Cassola, Moravia, Ottieri, Pasolini, Pratolini, Roversi, Volponl. Milano, Garzanti, 1973.

CAMON, Ferdinando. Letteratura e classi subalterne. Venezia-Padova, Marsilio Editori, 1974, pp. 41-47, 49-53.

LUPERINI, Romano. II Novecento. (1omo II). Torino, J_oescher, 1981.

BER'TACCHINI, Renato. Novecento. Molena, Ed. La Vela, 1982.

MANACORDA, Giuliano. Vent'anni di pazienza. Saggi sulla letteratura italiana contemporanea. Firenze, La Nuova Italia, 1972.

BÀRBERI SQUAROTTI, Giorgio. Poesin e narrativa del secondo Novecento. Milano, Mursia, 1971.

Abstract: In Le mosche del capllale, Volponi analyses the relationship between the capitalistic power and the industrial world. The novelist believes that literature may exert an influence on the class-warfare.

Key-words: political novel, capilalism and industry, contemporary fiction. 